\title{
Physics-based preconditioners for solving PDEs on highly heterogeneous media
}

\author{
Burak Aksoylu ${ }^{* 1}$ and Hector Klie $* * 2$ \\ ${ }^{1}$ Louisiana State University, Department of Mathematics and Center for Computation and Technology, \\ Baton Rouge, LA 70803, USA. \\ 2 The University of Texas at Austin, The Institute of Computational and Engineering Sciences, Austin, TX 78705, USA.
}

\begin{abstract}
Eigenvalues of smallest magnitude become a major bottleneck for iterative solvers especially when the underlying physical properties have severe contrasts. These contrasts are commonly found in many applications such as composite materials, geological rock properties, and thermal and electrical conductivity. The main objective of this work is to construct a method as algebraic as possible that could efficiently exploit the connectivity of highly heterogeneous media in the solution of diffusion operators. We propose an algebraic way of separating binary-like systems according to a given threshold into high- and low-conductivity regimes of coefficient size $O(m)$ and $O(1)$, respectively where $m \gg 1$. The condition number of the linear system depends both on the mesh size and the coefficient size $m$. For our purposes, we address only the $m$ dependence since the condition number of the linear system is mainly governed by the high-conductivity subblock. Thus, the proposed strategy is inspired by capturing the relevant physics governing the problem. Based on the algebraic construction, a two-stage preconditioning strategy is developed as follows: (1) a first stage that comprises approximation to the components of the solution associated to small eigenvalues and, (2) a second stage that deals with the remaining solution components with a deflation strategy (if ever needed). The deflation strategies are based on computing near invariant subspaces corresponding to smallest eigenvalues and deflating them by the use of recycled the Krylov subspaces. More detail on the proposed preconditioners can be found in [1].
\end{abstract}

\section{The preconditioner and exploiting the physics in the preprocessing step}

The main objective of the present work is to introduce a novel physics-based preconditioning strategy for solving problems with high physical contrasts in highly heterogeneous media applications. In porous media applications, these stringent situations commonly arise, for example, in multilayered geological formations composed of different type of rocks. We restrict our presentation with porous media flow, but generalizations to heterogeneous media easily follow. We assume that the porous media consist of highly permeable interconnected regions allowing for a strong global flow conductivity (e.g., channelized media). Figure 1 illustrates this type of permeability distribution settings that we are interested in handling efficiently from the iterative solution standpoint. The matrices under investigation correspond to finite volume discretization of the diffusion equation with the given permeability fields, and hence, they are symmetric positive definite, diagonally dominant, and highly ill-conditioned. We propose the following algorithm:

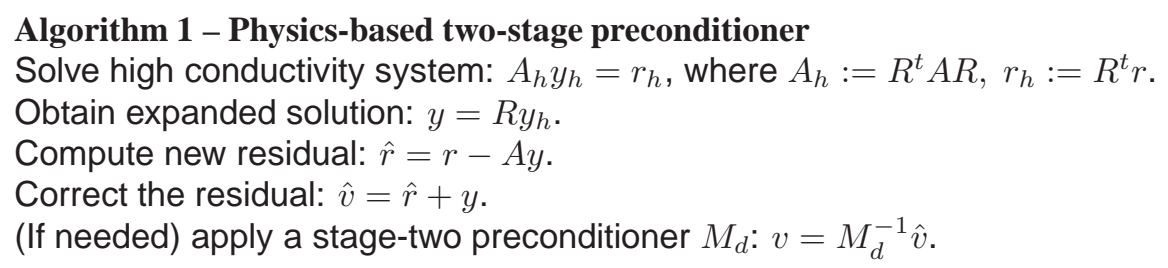

Before Algorithm 1, there is a preprocessing step which creates an ordering of the degrees of freedom (DOF) according to permeability contrasts. We define a threshold permeability value $\langle K\rangle$. DOF with permeability value larger than $\langle K\rangle$ are ordered first and those with a lower value are ordered after. This gives a $2 \times 2$ block formulation of the system matrix where DOF associated to high $(O(m))$ and low $(O(1))$ permeability values reside in $A_{h}^{\text {orig }}$ and $A_{l}^{\text {orig }}$, respectively.

Diagonal scaling adds a new dimension to the understanding of the effects permeability contrasts. Let $A:=D^{\text {orig }}{ }^{-1} A^{\text {orig }}$ and $A_{h}:=D_{h}^{\text {orig }^{-1}} A_{h}^{\text {orig }}$. In a stratified reservoir, diagonal scaling reveals that the permeability contrasts give rise to eigenvalues of smallest magnitude. The number of high permeable regions in the reservoir that are sandwiched by low permeable region gives the exact the number of smallest eigenvalues [2,3,4]. Therefore, $A_{h}$ contains vital information and can capture the main features of $A$ supported by our permeability based assumption. Most importantly, $A_{h}$ can capture smallest eigenvalues of $A$ which seem to be the main source of ill-conditioning. We end up with $A_{h}$ which is ill-conditioned but very small in

\footnotetext{
* Corresponding author: e-mail: burak@cct.Isu.edu, Phone: +1 225578 8984, Fax: +1 2255785362

** e-mail: klie@ices.utexas.edu, Phone: +1 512475 8634, Fax: +1 5122322445.
} 
size. Further ordering can be applied to DOF in $A_{h}$ if there is still extra variation in the high permeability values. This makes the size of $A_{h}$ even smaller, hence, its system solve easier. For instance, deflation methods or direct solvers can be used for $A_{h}$. Considering the below decompositions, we can relate Algorithm 1 to a matrix.

$$
A=\left\{\left[\begin{array}{ll}
I_{h} & 0 \\
A_{l h} A_{h}^{-1} & I_{l}
\end{array}\right]\left[\begin{array}{ll}
A_{h} & 0 \\
0 & I_{l}
\end{array}\right]\right\} \quad\left[\begin{array}{ll}
I_{h} & 0 \\
0 & A_{S}
\end{array}\right]\left[\begin{array}{ll}
I_{h} & A_{h}^{-1} A_{h l} \\
0 & I_{l}
\end{array}\right],
$$

The action of Algorithm 1 defines the left preconditioner in (2) which corresponds to the decomposition (1). An equivalent right preconditioner can also be formed.

$$
M_{\text {left }}^{-1}=\left[\begin{array}{ll}
A_{h}^{-1} & 0 \\
-A_{l h} A_{h}^{-1} & I_{l}
\end{array}\right], M_{\text {right }}^{-1}=\left[\begin{array}{ll}
A_{h}^{-1} & -A_{h}^{-1} A_{h l} \\
0 & I_{l}
\end{array}\right] .
$$

Then, we see that;

$$
\sigma\left(M_{\text {left }}^{-1} A\right)=\sigma\left(A M_{\text {right }}^{-1}\right)=\sigma\left(A_{S}\right) \cup\{1\} .
$$

If smallest eigenvalues are well captured by $A_{h}$, we observe that the Schur complement $A_{S}$ is free from smallest eigenvalues, and by (3), so is the preconditioned system (see the left pair in Figure 1). If not (see the right pair in Figure 1), we employ a deflation method as stage-two preconditioner on top of $M_{\text {left }}^{-1}$ and we show that this strategy is effective.

\section{Incorporating deflation as a stage-two preconditioner}

A typical deflation operator is designed to process the extremal eigenvalues in such a way that the resulting operator will a have better condition number in general. Let $U \in \mathbb{C}^{n \times r}$ be the exact invariant subspace corresponding to $r$ smallest eigenvalues. One type of deflation operator-is utilized as the stage-two preconditioner — that shifts the $r$ smallest eigenvalues to $\left|\lambda_{\max }(A)\right|$ and leaves the rest of the spectrum unchanged is given by: $C^{-1}=\left|\lambda_{\max }(A)\right| U\left(U^{T} A U\right)^{-1} U^{T}+\left(I-U U^{T}\right)$.

Deflation methods can be classified as static or dynamic. In static deflation, the deflation operator is determined before the iteration process starts and remains fixed throughout. In the dynamic version, the deflation operator is regularly updated as the fresh Krylov subspace information is computed. We utilize GMRES $(m)$ and dynamic deflation methods become attractive because they have the capability to exploit useful information in the Hessenberg matrix at each restart. We compare our preconditioner to three well-known deflation methods: harmonic [5], augmented [7], and Burrage-Erhel [6]. These are dynamic deflation methods where the near invariant subspace $U$ is extracted by a harmonic Ritz projection from the Hessenberg matrix. We report that our preconditioners outperform all of the three methods.
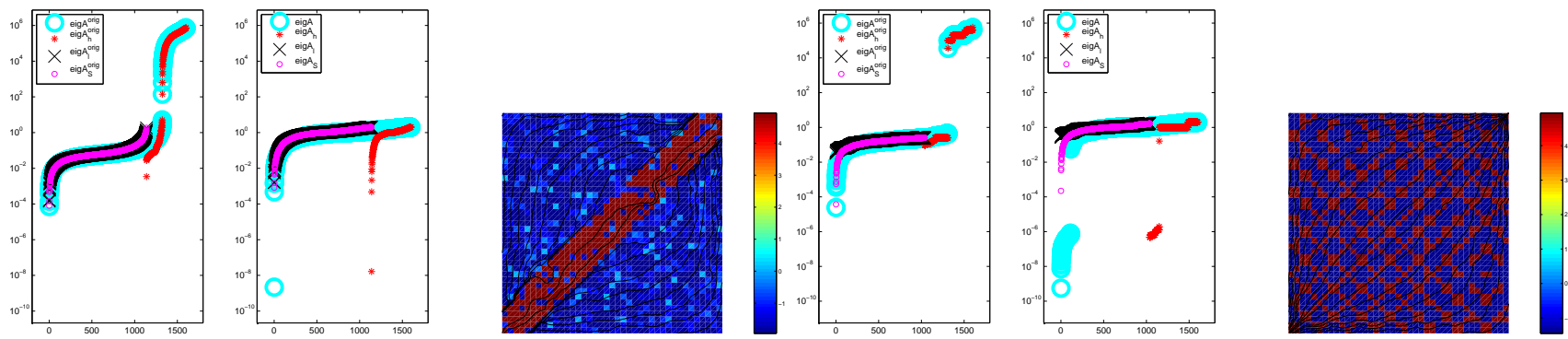

Fig. 1 Spectra and the corresponding log permeability fields. Streamlines indicate preferential flow paths. In the left pair with single channel geometry, notice that $A_{h}$ captures the smallest eigenvalue of $A$. Hence, the Schur complement $A_{S}$ does not contain small eigenvalues, whereas, this behaviour of $A_{h}$ cannot be achieved in the right pair with the checker-board like reservoir geometry.

\section{References}

[1] B. Aksoylu and H. Klie, Pysics-based Preconditioners for Solving PDEs on Highly Heterogeneous Media, Louisiana State University Center for Computation and Technology (CCT) Technical Report 07-01.

[2] I. G. Graham and M. J. Hagger, Unstructured additive Schwarz-conjugate gradient method for elliptic problems with highly discontinuous coefficients, SIAM J. Sci. Comp., 20, 2041-2066 (1999).

[3] C. Vuik, A.Segal, and J. Meijerink, An efficient preconditioned CG method for the solution of a class of layered problems with extreme contrasts of coefficients, J. of Comp. Phys., 152, 385-403 (1999).

[4] C. Vuik, A.Segal, J. Meijerink, and G.T.Wijma, The construction of projection vectors for a ICCG method applied to problems with extreme contrasts in the coefficients, J. of Comp. Phys., 172), 426-450 (2001.

[5] J. Erhel, K. Burrage, and B. Pohl, Restarted GMRES preconditioned by deflation, J. of Comput. and Appl. Math., 69, 303-318 (1996)

[6] K. Burrage and J. Erhel, On the performance of various adaptive preconditioned GMRES strategies, Numer. Linear Alg. Appl., 5. 101-121 (1998).

[7] R. B. Morgan, A Restarted GMRES Method augmented with eigenvectors, SIAM J. Matrix Anal. Appl., 16, 1154-1171 (1995). 